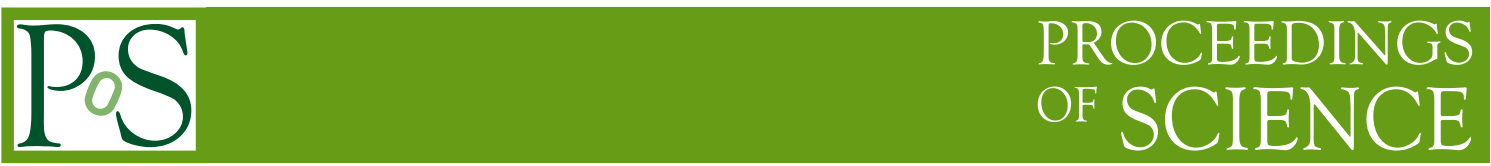

\title{
Non-geometric fluxes and non-associative geometry
}

\author{
Erik Plauschinn* \\ Institute for Theoretical Physics and Spinoza Institute \\ Utrecht University \\ 3508 TD Utrecht, The Netherlands \\ E-mail: e.plauschinn@uu.nl
}

\begin{abstract}
In these proceedings, we discuss non-commutativity in closed string theory. In analogy to the open-string sector, for closed strings we first motivate a cyclic double commutator to be evaluated for backgrounds with geometric or non-geometric fluxes. A non-trivial result for such an expression indicates a non-associative structure. Second, we define a conformal field theory at linear order in background fluxes and compute correlation functions therein. From these we motivate a tri-product which captures non-commutative and non-associative effects.
\end{abstract}

Proceedings of the Corfu Summer Institute 2011 School and Workshops on Elementary Particle Physics and Gravity

September 4-18, 2011

Corfu, Greece

Report numbers: SPIN-12/09 and ITP-UU-12/10

${ }^{*}$ Speaker. 


\section{Introduction and Motivation}

One of the remarkable features of string theory is that it provides a framework to treat gauge theories and gravity in a unified way, and which is expected to be complete in the ultra-violet (UV). Concerning gauge theories in field theory, it is known how to deal with (certain classes of) thereof in the UV via the procedure of renormalization. For gravity on the other hand, that question is understood to a far lesser degree. Although, one may expect that a theory of quantum gravity is related to a space-time which is non-commutative. In view of this expectation, also string theory should feature a non-commutative behavior.

However, non-commutativity in string theory was first discovered for D-branes which correspond to the gauge theory sector. More concretely, for an open string ending on a D-brane endowed with a gauge flux, the commutator of two open-string coordinates on the brane is generically nonvanishing $[1,2,3]$. Moreover, correlation functions of vertex operators on the D-brane also indicate a non-commutative structure which is encoded in a phase factor. Constructing then an effective action out of these correlators, it turns out that the phase factor can be incorporated via a Moyal-Weyl star product between the fields. One therefore obtains a non-commutative gauge theory on the D-brane [4].

For the gravity sector in string theory, non-commutativity appears to be harder to obtain (for earlier work on that subject see for instance [5]). But, given the results for the open string, a guideline to study the closed string may be to parallel the discussion of the former. Let us therefore highlight the following three points:

1. For the open string, as will be explained in more detail below, the non-commutativity parameter is related to the gauge flux on the D-brane. Therefore, in the closed-string sector we should consider backgrounds with non-trivial fluxes.

2. A quantity which clearly shows the non-commutative behavior for the open string is the commutator of two coordinates on the D-brane. For the closed string, we thus seek for a similar expression.

3. Correlation functions of open-string vertex operators exhibited a non-commutative behavior, which is related to the Moyal-Weyl star product. Therefore, also in the closed-string sector we should compute correlation functions and try to extract a non-commutative product.

Background fluxes Let us continue with a brief discussion of background fluxes. As just mentioned, for the open string the non-commutativity parameter is related to the gauge flux on the D-brane. For the closed string, the authors in [6] thus considered a background with non-vanishing $H$-flux realized via Wess-Zumino-Witten model [7]. Other flux backgrounds with geometric flux $f$ and non-geometric fluxes $Q$ and $R$ can be obtained by applying successive T-dualities $[8,9]$

$$
H_{x y z} \stackrel{T_{z}}{\longleftrightarrow} f_{x y}{ }^{2} \stackrel{T_{y}}{\longleftrightarrow} Q_{x}^{y z} \stackrel{T_{x}}{\longleftrightarrow} R^{x y z} .
$$

For the setting in [6], the most interesting situations were non-vanishing $H$-and $R$-fluxes, where the latter is expected to be related to a non-associative structure $[10,11]$. However, the question of non-commutativity in closed string theory was also studied in [12], where the cases of $H$-flux and geometric flux were analyzed in the framework of doubled geometry, leading to similar findings as in [6]. 


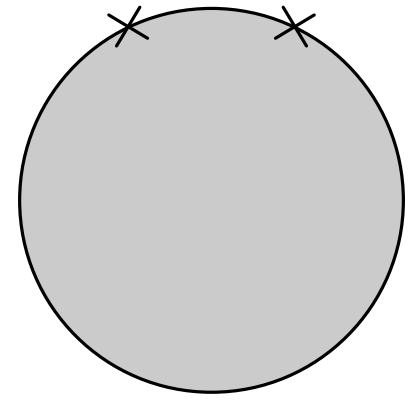

(a) Open string disc correlator with two vertex operators

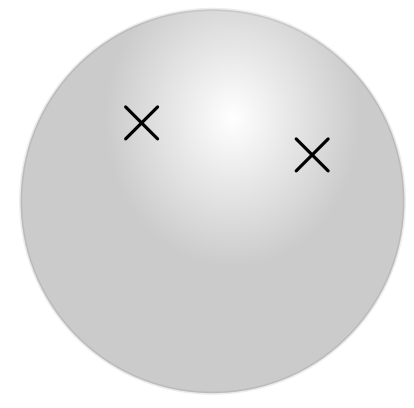

(b) Closed string correlator on the sphere with two vertex operators

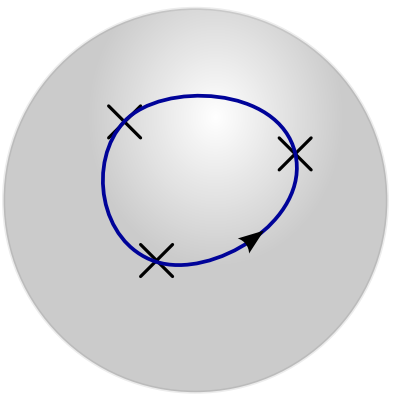

(c) Closed string correlator on the sphere with three vertex operators

Figure 1: Open string disc diagram (a) with two vertex operators, for which an ordering can be defined (locally). For two vertex operators on the sphere (b), no such ordering can be defined. For three vertex operators on the sphere (c), a line connecting the three insertion points has an orientation (locally).

To summarize, in order to investigate non-commutativity for the closed string, recent work suggests to consider backgrounds with non-vanishing $H$-flux as well as non-vanishing geometric or non-geometric fluxes.

Three-bracket We now turn to the question of how to identify a suitable expression displaying non-commutative behavior. For the open string, the origin of the non-commutativity can be explained heuristically in the following way. To compute the two-point function on the D-brane, as illustrated in figure 1(a), vertex operators are inserted at the boundary of a disc. Provided there is a quantity sensitive to the ordering of these operators, such as the gauge flux on the brane, the operators do not commute. For the closed string, the situation is different. Here, the world-sheet is a sphere rather than a disc, and vertex operators are inserted in the bulk of the sphere. As illustrated in figure 1(b), no ordering of two operators on the sphere can be defined. However, when inserting three vertex operators and connecting them through a closed line, as shown in figure 1(c), a different orientation of the line may be detected by a quantity sensitive to such a change.

This heuristic observation suggests that in order to observe non-commutativity for the closed string, one should consider three instead of two objects. In terms of commutators of closed string coordinates, a natural guess for such an expression is

$$
\left[X^{\mu}, X^{v}, X^{\rho}\right]:=\lim _{\sigma_{i} \rightarrow \sigma}\left[\left[X^{\mu}\left(\sigma_{1}, \tau\right), X^{v}\left(\sigma_{2}, \tau\right)\right], X^{\rho}\left(\sigma_{3}, \tau\right)\right]+\text { cyclic }
$$

Note that if this cyclic double commutator is non-vanishing, it indicates not only a non-commutative but also a non-associative structure (see also [13] within the proceedings of this conference).

Another argument supporting (1.2) is that for two closed-string coordinates $X^{\mu}(\sigma, \tau)$ the single commutator generically depends on the coordinates $\sigma$ and $\tau$ of the world-sheet. Therefore, such a quantity can not be expected to characterize a feature of the target space.

Correlation functions Non-commutative behavior may also be encoded in correlation functions of vertex operators, and so we briefly recall the situation for the open string. With $T_{i}$ denoting a tachyon vertex operator of the open bosonic string, $p_{i}$ the corresponding momentum, $\theta$ a flux 
parameter and $\varepsilon(\tau)=\operatorname{sign}(\tau)$, a correlation function of $N$ such operators is found to be

$$
\left\langle T_{1} \ldots T_{N}\right\rangle=\exp \left(i \sum_{1 \leq n<m \leq N} p_{n, a} \theta^{a b} p_{m, b} \varepsilon\left(\tau_{n}-\tau_{m}\right)\right) \times\left\langle T_{1} \ldots T_{N}\right\rangle_{\theta=0},
$$

which contains an extra phase due to the non-commutative nature of the theory. One can then define an $N$-product $\star_{N}$ in the following way

$$
\begin{aligned}
f_{1}(x) \star_{N} f_{2}(x) \star_{N} \ldots \star_{N} f_{N}(x):= \\
\left.\quad \exp \left(i \sum_{1 \leq n<m \leq N} \theta^{a b} \partial_{a}^{x_{n}} \partial_{b}^{x_{m}}\right) f_{1}\left(x_{1}\right) f_{2}\left(x_{2}\right) \ldots f_{N}\left(x_{N}\right)\right|_{x_{1}=\ldots=x_{N}=x},
\end{aligned}
$$

which correctly reproduces the phase appearing in (1.3). Note that these $N$-products are related to the subsequent application of the usual star-product $\star=\star_{2}$. Therefore, by evaluating correlation functions of vertex operators in open string theory, it is possible to derive the Moyal-Weyl product and some of its features [4]. Moreover, in [14] and [15] this product has been studied in the context of the open string, and a non-associative behavior has been found.

By analogy, for the closed string we then also have to compute correlation functions and identify the dependence on the flux. If the latter can be encoded in a phase factor, an analysis along similar lines as for the open string can be performed. Although quite ambitious, from these correlation functions a product like the Moyal-Weyl star product may be extracted, and a theory of quantum gravity using such a product may be formulated.

Strategy and outline for these proceedings The points alluded to above suggest that to study non-commutativity for the closed string, a cyclic double commutator in a background with nonvanishing fluxes should be computed. A convenient setting for such an analysis are Wess-ZuminoWitten models, which we employ in section 2 to compute (1.2) for the coordinates of the closed bosonic string. Two of the main results will be that a non-commutative behavior for the closed string can indeed be seen, and that this feature appears already at linear order in the flux parameter.

However, since non-commutativity can also be detected in correlation functions, in section 3 we define a conformal field theory at linear order in the flux and introduce vertex operators. Computing then correlation functions of the latter and studying the phase factor of these expressions, we are led to the definition of a tri-product. One might suspect that this product can be used to construct a non-commutative theory of gravity, which, however, has not been achieved to date.

As a final remark, let us mention that these proceedings cover a talk given some time ago and so further developments and new results have appeared. In particular, as mentioned above, non-commutativity in closed string theory has also been studied in [12], and examples have been constructed in [16]. A comprehensive summary of the ideas in [6] and [17], with a different emphasis compared to here, can be found in the proceedings [18]. Furthermore, as we pointed out above, backgrounds with $H$-, geometric and non-geometric fluxes are important for the question of closed-string non-commutativity. Recently, such settings have been studied for instance in [19] from the point of generalized complex geometry, and in [20] in the framework of double field theory (see also [21]). Furthermore, in [22] a Palatini formulation of (higher-order) Lovelock gravity has been analyzed, and requiring consistency of the Palatini formalism lead to constraints which can be interpreted as Bianchi identities for geometric and non-geometric fluxes. This analysis will be continued in [23]. 


\section{Closed string non-commutativity}

In this section we approach the question about non-commutativity for closed strings. In the open-string sector, non-commutativity appeared for strings ending on D-branes endowed with a background flux $[1,2,3]$. In the following, we will therefore first briefly review the analysis in the open-string case, and then perform a similar computation for closed strings. This section is based on [6], where further details can be found.

\subsection{Open string non-commutativity}

In this subsection, we mainly follow the discussion in [2].

Open string computation We consider an open string with both endpoints on a $\mathrm{D} p$-brane carrying constant two-form flux $\mathscr{F}_{i j}=B_{i j}+F_{i j}$, where $i, j=0, \ldots, p$. This leads to mixed NeumannDirichlet boundary conditions longitudinal to the brane, so that the mode expansions for the corresponding free bosons read

$$
X^{i}(\sigma, \tau)=x_{0}^{i}+\left(\alpha_{0}^{i} \tau-\alpha_{0}^{j} \mathscr{F}_{j}{ }^{i} \sigma\right)+\sum_{n \neq 0} \frac{e^{-i n \tau}}{n}\left(i \alpha_{n}^{i} \cos (n \sigma)-\alpha_{n}^{j} \mathscr{F}_{j}{ }^{i} \sin (n \sigma)\right) .
$$

Here we normalized $0 \leq \sigma \leq \pi$, and indices of $\mathscr{F}_{i j}$ are raised by the inverse metric of the form $\eta^{i j}=$ $\operatorname{diag}(-1,+1, \ldots,+1)$. As carried out in [2], the commutation relations for the modes appearing in (2.1) can be obtained via canonical quantization. Using these relations, the equal-time commutator is evaluated as

$$
\left[X^{i}\left(\sigma_{1}, \tau\right), X^{j}\left(\sigma_{2}, \tau\right)\right]=-2 i \alpha^{\prime}\left(M^{-1} \mathscr{F}\right)^{i j}\left[P\left(\sigma_{1}, \sigma_{2}\right)+\sum_{n \neq 0} \frac{\sin n\left(\sigma_{1}+\sigma_{2}\right)}{n}\right],
$$

where $M_{i j}=\delta_{i j}-\mathscr{F}_{i}^{k} \mathscr{F}_{k j}$ and matrix products are understood. The function $P$ is a continuous linear expression in the world-sheet coordinates $\sigma_{i}$ of the form

$$
P\left(\sigma_{1}, \sigma_{2}\right)=\sigma_{1}+\sigma_{2}-\pi
$$

which arises purely from the commutation relations involving the zero modes $x_{0}^{i}$ and $\alpha_{0}^{i}$. The sum in (2.2) originates from the oscillator modes $\alpha_{n}^{i}$ for $n \neq 0$, and can be further evaluated using the Fourier transform

$$
\gamma(\varphi)=\sum_{n=1}^{\infty} \frac{\sin (n \varphi)}{n}=\left\{\begin{array}{cl}
\frac{1}{2}(\pi-\varphi) & 0<\varphi<2 \pi \\
0 & \varphi=0,2 \pi
\end{array}\right.
$$

Using then equations (2.3) and (2.4), we see that for $0<\sigma_{1}+\sigma_{2}<2 \pi$ the two terms in (2.2) cancel. However, on the boundaries $\sigma_{1}=\sigma_{2}=0$ and $\sigma_{1}=\sigma_{2}=\pi$ one obtains

$$
\left[X^{i}(0, \tau), X^{j}(0, \tau)\right]=-\left[X^{i}(\pi, \tau), X^{j}(\pi, \tau)\right]=2 \pi i \alpha^{\prime}\left(M^{-1} \mathscr{F}\right)^{i j}
$$

In summary, the equal-time, equal-position commutator between two target-space coordinates $X^{i}(\sigma, \tau)$ does not vanish along a D-brane carrying non-trivial two-form flux $\mathscr{F}_{i j}$. 
Remarks Let us make the following remarks:

- Even without knowing the zero mode contribution $P\left(\sigma_{1}, \sigma_{2}\right)$ explicitly, we could have guessed this function by requiring the commutator (2.2) to vanish for generic points on the world-sheet. In turn, the non-zero result in (2.5) arises from the boundaries of the open string due to the discontinuity of $\gamma(\varphi)$ at $\varphi=0 \bmod 2 \pi$.

- Since the equal-time, equal-position commutator (2.5) is independent of the world-sheet coordinates $\sigma$ and $\tau$, one can indeed conclude that this world-sheet computation reveals a feature of the target space (as probed by an open string).

\subsection{Closed string non-commutativity}

For closed strings, we expect non-commutativity to arise for backgrounds with a non-vanishing flux. As a consequence, the equations of motion require the space to be curved. One of the simplest examples for an exactly solvable configuration are Wess-Zumino-Witten (WZW) [7] models describing compactifications with $H$-flux [24].

Wess-Zumino-Witten models Let us therefore start our discussion by considering the WZW model for the group manifold $S U(2)$. The corresponding action is given by

$$
\begin{aligned}
S= & \frac{k}{16 \pi} \int_{\partial \Sigma} d^{2} x \operatorname{Tr}\left[\left(\partial_{\alpha} g\right)\left(\partial^{\alpha} g^{-1}\right)\right] \\
& -\frac{i k}{24 \pi} \int_{\Sigma} d^{3} y \varepsilon^{\tilde{\alpha} \tilde{\beta} \tilde{\gamma}} \operatorname{Tr}\left[\left(g^{-1} \partial_{\tilde{\alpha}} g\right)\left(g^{-1} \partial_{\tilde{\beta}} g\right)\left(g^{-1} \partial_{\tilde{\gamma}} g\right)\right],
\end{aligned}
$$

where $k \in \mathbb{Z}^{+}$denotes the level and $\Sigma$ is a three-dimensional manifold with boundary $\partial \Sigma$. The indices take values $\alpha=1,2$ and $\tilde{\alpha}, \ldots=1,2,3$, which are raised or lowered by the metrics $h_{\alpha \beta}=$ $\operatorname{diag}(+1,+1)$ and $h_{\tilde{\alpha} \tilde{\beta}}=\operatorname{diag}(+1,+1,+1)$, respectively. Parametrizing an element $g \in S U(2)$ in terms of Hopf coordinates $\eta^{i}$ as

$$
g=\left(\begin{array}{cc}
e^{i \eta^{2}} \cos \eta^{1} & e^{i \eta^{3}} \sin \eta^{1} \\
-e^{-i \eta^{3}} \sin \eta^{1} & e^{-i \eta^{2}} \cos \eta^{1}
\end{array}\right),
$$

with $0 \leq \eta^{1} \leq \pi / 2,0 \leq \eta^{2,3} \leq 2 \pi$, one realizes that the first term in (2.6) is a non-linear sigma model with target space $S^{3}$ of radius $R=\sqrt{k}$, and the second term corresponds to a background flux proportional to $k$.

Conserved currents and Kac-Moody algebras Solving the model (2.6) directly in terms of Hopf coordinates $\eta^{i}$ is not easily possible, but it is well known that the WZW model actually is exactly solvable. To see this, we introduce a complex coordinate $z=\exp \left(x^{1}+i x^{2}\right)$ and define the currents

$$
J=J^{a} \frac{\sigma^{a}}{\sqrt{2}}=-k\left(\partial_{z} g\right) g^{-1}, \quad \bar{J}=\bar{J}^{a} \frac{\sigma^{a}}{\sqrt{2}}=+k g^{-1}\left(\partial_{\bar{z}} g\right) .
$$

Note that here and in the following, $\sigma^{a}$ with $a=1,2,3$ are the Pauli matrices and summation over repeated indices is understood. From the equation of motion of the WZW model (2.6) it follows 
that the currents $J^{a}$ are holomorphic and that the $\bar{J}^{a}$ are anti-holomorphic. Therefore, one can perform the Laurent expansions

$$
J^{a}(z)=\sum_{n \in \mathbb{Z}} j_{n}^{a} z^{-n-1}, \quad \bar{J}^{a}(\bar{z})=\sum_{n \in \mathbb{Z}} \bar{j}_{n}^{a} \bar{z}^{-n-1} .
$$

The symmetry transformations of the WZW model then translate into the following commutation relations for the modes $j_{n}^{a}$ and $\bar{j}_{n}^{a}$

$$
\begin{aligned}
& {\left[j_{m}^{a}, j_{n}^{b}\right]=i f^{a b}{ }_{c} j_{m+n}^{c}+k m \delta_{m+n} \delta^{a b},} \\
& {\left[\bar{j}_{m}^{a}, \bar{j}_{n}^{b}\right]=i f_{c}^{a b} \bar{j}_{m+n}^{c}+k m \delta_{m+n} \delta^{a b},}
\end{aligned}
$$$$
\left[j_{m}^{a}, \bar{j}_{n}^{b}\right]=0
$$

which define two independent Kac-Moody algebras. Note that the structure constants for $S U(2)$ in our convention read $f^{a b c}=\sqrt{2} \varepsilon^{a b c}$, and indices are raised or lowered by $\delta^{a b}$ and $\delta_{a b}$, respectively. For later reference, let us then employ the parametrization (2.7) in (2.8) and express the two currents (2.9) as follows

$$
J^{a}(z)=-i \sqrt{2} k \mathrm{E}_{i}^{a} \partial_{z} \eta^{i}, \quad \bar{J}^{a}(\bar{z})=-i \sqrt{2} k \overline{\mathrm{E}}_{i}^{a} \partial_{\bar{z}} \eta^{i} .
$$

The matrices $\mathrm{E}^{a}{ }_{i}$ and $\overline{\mathrm{E}}^{a}{ }_{i}$ are known explicitly (see [6]), and depend on the coordinates $\eta^{i}$.

Local coordinates So far, we have mainly reviewed the well-known geometry for the exactly solvable $S U(2)_{k}$ WZW model. However, let us now introduce fields $X^{a}(z, \bar{z})$ according to

$$
\begin{aligned}
& J^{a}(z)=-i \sqrt{k} \partial_{z} X^{a}(z, \bar{z})=-i \sqrt{2} k \mathrm{E}^{a}{ }_{i}(\vec{\eta}) \partial_{z} \eta^{i}(z, \bar{z}), \\
& \bar{J}^{a}(z)=-i \sqrt{k} \partial_{\bar{z}} X^{a}(z, \bar{z})=-i \sqrt{2} k \overline{\mathrm{E}}^{a}{ }_{i}(\vec{\eta}) \partial_{\bar{z}} \eta^{i}(z, \bar{z}) .
\end{aligned}
$$

It is clear that the $X^{a}$ do not correspond to bona fide global coordinates on $S^{3}$ since there does not exist a flat metric on $S^{3}$. However, as shown in [25], if the $X^{a}(z, \bar{z})$ satisfy their (free) equations of motion, the $\eta^{i}(z, \bar{z})$ do so as well.

Next, since the cyclic double commutator (1.2) we are interested in is a local quantity, we can imagine to probe the geometry around a point $\vec{\eta}_{0}$ on a three-sphere $S^{3}$ by a closed string. Writing then

$$
X^{a}(z, \bar{z})=X^{a}(z)+\bar{X}^{a}(\bar{z})
$$

and using (2.12), locally we can identify the left- and right-moving coordinates as

$$
X^{a}(z) \simeq \sqrt{2 k} \mathrm{E}^{a}{ }_{i}\left(\vec{\eta}_{0}\right) \eta^{i}(z), \quad \bar{X}^{a}(\bar{z}) \simeq \sqrt{2 k} \overline{\mathrm{E}}^{a}{ }_{i}\left(\vec{\eta}_{0}\right) \bar{\eta}^{i}(\bar{z})
$$

The mode expansions of $X^{a}(z)$ and $\bar{X}^{a}(\bar{z})$ are found by integrating the expansions of the currents given in (2.9). In particular, for the holomorphic part we arrive at

$$
X^{a}(z)=\frac{i}{\sqrt{k}} x_{0}^{a}-\frac{i}{\sqrt{k}} j_{0}^{a} \log z+\frac{i}{\sqrt{k}} \sum_{n \neq 0} \frac{j_{n}^{a}}{n} z^{-n},
$$

and a similar expression is obtained for the anti-holomorphic part $\bar{X}^{a}(\bar{z})$. The modes $j_{n}^{a}$ in (2.15) satisfy the corresponding Kac-Moody algebra given in (2.10), however, a priori it is not clear what the precise form of the commutation relations involving $x_{0}^{a}$ is. In the following, we are going to fix this contribution in analogy to the open string discussed in section 2.1 . 
Cyclic double commutator Let us consider the cyclic double commutator for the holomorphic part $X^{a}(z)$ of the free fields (2.13)

$$
\left[X^{a}\left(z_{1}\right), X^{b}\left(z_{2}\right), X^{c}\left(z_{3}\right)\right]=\left[\left[X^{a}\left(z_{1}\right), X^{b}\left(z_{2}\right)\right], X^{c}\left(z_{3}\right)\right]+\text { cyclic }
$$

evaluated at equal times. For our choice of complex coordinates $z_{i}=\exp \left(\tau_{i}+i \sigma_{i}\right)$ this implies $\left|z_{1}\right|=\left|z_{2}\right|=\left|z_{3}\right|$, which will always be understood for the expression (2.16). To simplify the following formulae, let us furthermore introduce $\mathbf{x}^{a}, \mathbf{p}^{a}$ and $\mathbf{j}^{a}$ as

$$
\mathbf{x}^{a}=\frac{i}{\sqrt{k}} x_{0}^{a}, \quad \mathbf{p}^{a}(z)=-\frac{i}{\sqrt{k}} j_{0}^{a} \log z, \quad \mathbf{j}^{a}(z)=\frac{i}{\sqrt{k}} \sum_{n \neq 0} \frac{j_{n}^{a}}{n} z^{-n} .
$$

For the computation of (2.16), we first collect all terms involving $\mathbf{x}^{a}$ into a so far undetermined function $\mathscr{P}^{a b c}$

$$
\mathscr{P}^{a b c}\left(z_{1}, z_{2}, z_{3}\right)=\left[\mathbf{x}^{a}, \mathbf{x}^{b}, \mathbf{x}^{c}\right]+\left[\mathbf{x}^{a}, \mathbf{x}^{b}, \cdot\right]+\left[\mathbf{x}^{a}, \cdot, \cdot\right]+\ldots .
$$

For all other contributions in (2.16), we employ the Kac-Moody algebra (2.10) of the modes $j_{n}^{a}$ as well as the Jacobi identity for the structure constants $f^{a b}{ }_{c}$. Apart from (2.18), the only nonvanishing double commutator then reads

$$
\left[\mathbf{j}^{a}\left(z_{1}\right), \mathbf{j}^{b}\left(z_{2}\right), \mathbf{j}^{c}\left(z_{3}\right)\right]=-\frac{f^{a b c}}{\sqrt{k}} \sum_{\substack{n, m \neq 0 \\ n+m \neq 0}} \frac{1}{n m}\left(\frac{z_{3}}{z_{1}}\right)^{n}\left(\frac{z_{3}}{z_{2}}\right)^{m}+\text { cyclic } .
$$

Remember that this expression is understood to be evaluated at equal times. For the right-hand side in (2.19), we split the sums in the following way and compute

$$
\begin{aligned}
\Gamma\left(\sigma_{1}, \sigma_{2}, \sigma_{3}\right) & =-\sum_{n, m \neq 0} \frac{1}{n m}\left(\frac{z_{3}}{z_{1}}\right)^{n}\left(\frac{z_{3}}{z_{2}}\right)^{m}-\sum_{n \neq 0} \frac{1}{n^{2}}\left(\frac{z_{2}}{z_{1}}\right)^{n}+\text { cyclic } \\
& =\left\{\begin{array}{cl}
-\pi^{2} & \sigma_{1}=\sigma_{2}=\sigma_{3}, \\
0 & \text { else. }
\end{array}\right.
\end{aligned}
$$

Combining the above results, we arrive at the equal-time double commutator of the holomorphic fields $X^{a}(z)$ of the form

$$
\left[X^{a}\left(z_{1}\right), X^{b}\left(z_{2}\right), X^{c}\left(z_{3}\right)\right]=\mathscr{P}^{a b c}\left(z_{1}, z_{2}, z_{3}\right)+\frac{f^{a b c}}{\sqrt{k}} \Gamma\left(\sigma_{1}, \sigma_{2}, \sigma_{3}\right) .
$$

For the computation in the anti-holomorphic sector, we note that the modes $\bar{j}_{n}^{a}$ satisfy the same KacMoody algebra as $j_{n}^{a}$. Furthermore, we have $\bar{z}_{i}=e^{\tau_{i}-i \sigma_{i}}$ and so we only need to replace $\sigma_{i} \rightarrow-\sigma_{i}$ in the result (2.21) for the holomorphic sector. However, observe that the function $\Gamma$ is invariant under that substitution. Therefore, the result for the full equal-time double commutator reads

$$
\begin{aligned}
& {\left[X^{a}\left(z_{1}, \bar{z}_{1}\right), X^{b}\left(z_{2}, \bar{z}_{2}\right), X^{c}\left(z_{3}, \bar{z}_{3}\right)\right]} \\
& \quad=\mathscr{P}^{a b c}\left(z_{1}, z_{2}, z_{3}\right)+\overline{\mathscr{P}}^{a b c}\left(\bar{z}_{1}, \bar{z}_{2}, \bar{z}_{3}\right)+2 \frac{f^{a b c}}{\sqrt{k}} \Gamma\left(\sigma_{1}, \sigma_{2}, \sigma_{3}\right) .
\end{aligned}
$$

It is now tempting to follow the same logic as for the open-string computation. That is, we fix the unknown contribution $\mathscr{P}+\overline{\mathscr{P}}$ of the zero modes $x_{0}^{a}$ and $\bar{x}_{0}^{a}$ by: 
Assumption: $\quad$ The zero mode contribution $\mathscr{P}+\overline{\mathscr{P}}$ is continuous; and for the three points $z_{i}$ not all equal, the equal-time double commutator has to vanish.

More concretely, this assumption means that

$$
\mathscr{P}^{a b c}\left(z_{1}, z_{2}, z_{3}\right)+\overline{\mathscr{P}}^{a b c}\left(\bar{z}_{1}, \bar{z}_{2}, \bar{z}_{3}\right)=0 .
$$

Using then (2.20) and (2.23) in (2.22), we arrive at the following result

$$
\left[X^{a}, X^{b}, X^{c}\right]:=\lim _{z_{i} \rightarrow z}\left[X^{a}\left(z_{1}, \bar{z}_{1}\right), X^{b}\left(z_{2}, \bar{z}_{2}\right), X^{c}\left(z_{3}, \bar{z}_{3}\right)\right]=-\frac{2 \pi^{2}}{\sqrt{k}} f^{a b c} .
$$

Therefore, pursuing the same reasoning as for the open string, we are led to the intriguing result that the fields $X^{a}$ satisfy a non-vanishing three-bracket, where the right-hand side is constant and proportional to the $S U(2)$ structure constants $f^{a b c}$.

Summary and remarks Let us make the following remarks:

- In order to get a better understanding of the expression (2.24), we mention that for a fundamental product $x^{i} \bullet x^{j}$ one can define a three-bracket as

$$
\left[x^{1}, x^{2}, x^{3}\right]=\sum_{\sigma \in P_{3}} \operatorname{sign}(\sigma)\left(\left(x^{\sigma(1)} \bullet x^{\sigma(2)}\right) \bullet x^{\sigma(3)}-x^{\sigma(1)} \bullet\left(x^{\sigma(2)} \bullet x^{\sigma(3)}\right)\right),
$$

being the completely anti-symmetrized associator of this $\bullet$-product. For an associative product this expression vanishes, and so the non-vanishing result (2.24) indicates both a noncommutative and non-associative (NCA) structure.

- The equal-time, equal-position double commutator is independent of the world-sheet coordinates. Thus, it is expected to reflect a property of the target space (as probed by a closed string).

- Recalling that the radius of the three-sphere is $R=\sqrt{k}$, we realize that in the large radius limit $R \rightarrow \infty$ the NCA effect vanishes.

- We also computed the single commutator $\lim _{z_{i} \rightarrow z}\left[X^{a}\left(z_{1}, \bar{z}_{1}\right), X^{b}\left(z_{2}, \bar{z}_{2}\right)\right]$ and found it to be dependent on the world-sheet coordinates. We therefore conclude that the fundamental, welldefined target space structure is a three-bracket.

- As explained in more detail in [6], in contrast to the Hopf coordinates $\eta^{i}$ the fields $X^{a}(z, \bar{z})$ in (2.13) are not proper coordinates on the sphere. Therefore, the structure constants $f^{a b c}$ appearing on the right-hand side in (2.24) have to be interpreted properly. In particular, they should be interpreted as a non-geometric $R$-flux.

To summarize, denoting the flux-parameter by $\theta^{a b c}$, in the case of non-geometric $R$-flux the equaltime, equal-position cyclic double commutator indicates a non-associative structure and reads

$$
\left[X^{a}, X^{b}, X^{c}\right]:=\lim _{z_{i} \rightarrow z}\left[X^{a}\left(z_{1}, \bar{z}_{1}\right),\left[X^{b}\left(z_{2}, \bar{z}_{2}\right), X^{c}\left(z_{3}, \bar{z}_{3}\right)\right]\right]+\text { cycl. }=\theta^{a b c}
$$




\section{Correlation functions and tri-product}

In this section, we first define a conformal field theory (CFT) for the closed-string sector which captures effects at linear order in the background flux. Then, we compute correlation functions of vertex operators therein, and extract a non-commutative product. This part is based on [17], where more details can be found.

\subsection{Structure of the conformal field theory}

Setting Here, we do not start from an exactly solvable WZW model and then take a local limit, but rather from a flat background with flux. More concretely, our framework is that of a flat space with constant $H$-flux and dilaton which is to be considered as part of a full bosonic string theory construction. The metric and the flux are specified by

$$
d s^{2}=\sum_{a=1}^{N}\left(d X^{a}\right)^{2}, \quad H=\frac{2}{\alpha^{\prime 2}} \theta_{a b c} d X^{a} \wedge d X^{b} \wedge d X^{c}
$$

where in the following we focus mostly on $N=3$. A closed string moving in this background can be described by a sigma-model. With $\Sigma$ denoting the world-sheet of the closed string, its action reads

$$
\mathscr{S}=\frac{1}{2 \pi \alpha^{\prime}} \int_{\Sigma} d^{2} z\left(g_{a b}+B_{a b}\right) \partial X^{a} \bar{\partial} X^{b}
$$

where the metric for our particular situation is given by $g_{a b}=\delta_{a b}$, and for the $B$-field we choose a gauge in which $B_{a b}=\frac{1}{3} H_{a b c} X^{c}$.

Let us point out that already at lowest order in $\alpha^{\prime}$, the background given by (3.1) is not a solution to the string equations of motion. In particular, the beta-functional for the graviton

$$
\beta_{a b}^{G}=\alpha^{\prime} R_{a b}-\frac{\alpha^{\prime}}{4} H_{a}^{c d} H_{b c d}+2 \alpha^{\prime} \nabla_{a} \nabla_{b} \Phi+O\left(\alpha^{\prime 2}\right)
$$

does not vanish for (3.1) in the case of a constant dilaton $\Phi$. Only at linear order in the $H$-flux the above background provides a solution. We can thus conclude that the flat-space background with constant $H$ and $\Phi$ corresponds to a bona fide conformal field theory at linear order in the flux. Furthermore, since the three-bracket (2.26) is linear in $\theta^{a b c} \sim H^{a b c}$, up to first order in the $H$-flux we expect to find a reliable world-sheet CFT framework capturing potential non-associative effects.

Three-current correlators We continue by noting that the closed string coordinates $X^{a}(z, \bar{z})$ appearing in (3.2) are actually not proper conformal fields. Only the currents have a well-defined behavior under conformal transformations. Therefore, as usual, for the free theory we define

$$
J^{a}(z)=i \partial X^{a}(z), \quad \bar{J}^{a}(\bar{z})=i \bar{\partial} X^{a}(z)
$$

which at zeroth order in $H$ are indeed holomorphic and anti-holomorphic, respectively. Employing now the framework of conformal perturbation theory (see [17] for more details on the computation), 
for the correlators of three currents (3.4) (up to first order in the $H$-flux) we find

$$
\begin{aligned}
& \left\langle J^{a}\left(z_{1}\right) J^{b}\left(z_{2}\right) J^{c}\left(z_{3}\right)\right\rangle=-i \frac{\alpha^{\prime 2}}{8} H^{a b c} \frac{1}{z_{12} z_{23} z_{13}}, \quad\left\langle J^{a}\left(z_{1}\right) J^{b}\left(z_{2}\right) \bar{J}^{c}\left(\bar{z}_{3}\right)\right\rangle=-i \frac{\alpha^{\prime 2}}{8} H^{a b c} \frac{\bar{z}_{12}}{z_{12}^{2} \bar{z}_{23} \bar{z}_{13}}, \\
& \left\langle\bar{J}^{a}\left(\bar{z}_{1}\right) \bar{J}^{b}\left(\bar{z}_{2}\right) \bar{J}^{c}\left(\bar{z}_{3}\right)\right\rangle=+i \frac{\alpha^{\prime 2}}{8} H^{a b c} \frac{1}{\bar{z}_{12} \bar{z}_{23} \bar{z}_{13}}, \quad\left\langle\bar{J}^{a}\left(\bar{z}_{1}\right) \bar{J}^{b}\left(\bar{z}_{2}\right) J^{c}\left(z_{3}\right)\right\rangle=+i \frac{\alpha^{\prime 2}}{8} H^{a b c} \frac{z_{12}}{\bar{z}_{12}^{2} z_{23} z_{13}},
\end{aligned}
$$

where we made use of the anti-symmetry of $H_{a b c}$, raised the indices of $H$ with $\delta^{a b}$ and used $z_{i j}=$ $z_{i}-z_{j}$. As one can see, these expressions are not purely holomorphic or purely anti-holomorphic, but mixed terms appear. However, we have been using the currents (3.4) which are only valid for the free theory. To work at first order in the flux, we should take into account corrections to (3.4) linear in $H$. Let us therefore define new fields $\mathscr{J}^{a}$ and $\bar{J}^{a}$ in terms of (3.4) in the following way

$$
\mathscr{J}^{a}(z, \bar{z})=J^{a}(z)-\frac{1}{2} H_{b c}^{a} J^{b}(z) X_{R}^{c}(\bar{z}), \quad \bar{J}^{a}(z, \bar{z})=\bar{J}^{a}(\bar{z})-\frac{1}{2} H^{a}{ }_{b c} X_{L}^{b}(z) \bar{J}^{c}(\bar{z}) .
$$

For these, the only non-vanishing correlators of three fields (up to first order in the flux) are then either purely holomorphic or purely anti-holomorphic

$$
\begin{aligned}
& \left\langle\mathscr{J}^{a}\left(z_{1}, \bar{z}_{1}\right) \mathscr{J}^{b}\left(z_{2}, \bar{z}_{2}\right) \mathscr{J}^{c}\left(z_{3}, \bar{z}_{3}\right)\right\rangle=-i \frac{\alpha^{\prime 2}}{8} H^{a b c} \frac{1}{z_{12} z_{23} z_{13}}, \\
& \left\langle\overline{\mathscr{J}}^{a}\left(z_{1}, \bar{z}_{1}\right) \overline{\mathscr{J}}^{b}\left(z_{2}, \bar{z}_{2}\right) \overline{\mathscr{J}}^{c}\left(z_{3}, \bar{z}_{3}\right)\right\rangle=+i \frac{\alpha^{\prime 2}}{8} H^{a b c} \frac{1}{\bar{z}_{12} \bar{z}_{23} \bar{z}_{13}} .
\end{aligned}
$$

Furthermore, using the equation of motion derived from the action (3.2), at linear order in $H$ we compute $\bar{\partial} \mathscr{J}^{a}(z, \bar{z})=0$ and $\partial \overline{\mathscr{J}}^{a}(z, \bar{z})=0$, so these fields are indeed holomorphic and antiholomorphic, respectively. From now on, they will be denoted as $\mathscr{J}^{a}(z)$ and $\overline{\mathscr{J}}^{a}(\bar{z})$.

Current algebra and energy-momentum tensor Let us now study the fields $\mathscr{J}^{a}(z)$ and $\overline{\mathscr{J}}^{a}(\bar{z})$ in more detail. Their non-vanishing two-point function up to first order in $H$ is readily found to be

$$
\begin{aligned}
& \left\langle\mathscr{J}^{a}\left(z_{1}\right) \mathscr{J}^{b}\left(z_{2}\right)\right\rangle=\left\langle J^{a}\left(z_{1}\right) J^{b}\left(z_{2}\right)\right\rangle_{0}=\frac{\alpha^{\prime}}{2} \frac{1}{\left(z_{1}-z_{2}\right)^{2}} \delta^{a b}, \\
& \left\langle\bar{J}^{a}\left(\bar{z}_{1}\right) \bar{J}^{b}\left(\bar{z}_{2}\right)\right\rangle=\left\langle\bar{J}^{a}\left(z_{1}\right) \bar{J}^{b}\left(z_{2}\right)\right\rangle_{0}=\frac{\alpha^{\prime}}{2} \frac{1}{\left(\bar{z}_{1}-\bar{z}_{2}\right)^{2}} \delta^{a b},
\end{aligned}
$$

where we employed the definition (3.4) of the currents $J^{a}(z)$ as well as the two-point function of the fields $X^{a}(z, \bar{z})$. Taking then into account the three-point functions (3.7) of the fields $\mathscr{J}^{a}(z)$ and $\overline{\mathscr{J}}^{a}(\bar{z})$, with the help of (3.8) we can construct the following OPEs

$$
\begin{aligned}
& \mathscr{J}^{a}\left(z_{1}\right) \mathscr{J}^{b}\left(z_{2}\right)=\frac{\alpha^{\prime}}{2} \frac{\delta^{a b}}{\left(z_{1}-z_{2}\right)^{2}}-\frac{\alpha^{\prime}}{4} \frac{i H^{a b}{ }_{c}}{z_{1}-z_{2}} \mathscr{J}^{c}\left(z_{2}\right)+\text { reg. }, \\
& \bar{J}^{a}\left(\bar{z}_{1}\right) \overline{\mathscr{J}}^{b}\left(\bar{z}_{2}\right)=\frac{\alpha^{\prime}}{2} \frac{\delta^{a b}}{\left(\bar{z}_{1}-\bar{z}_{2}\right)^{2}}+\frac{\alpha^{\prime}}{4} \frac{i H^{a b}{ }_{c}}{\bar{z}_{1}-\bar{z}_{2}} \overline{\mathscr{J}}^{c}\left(\bar{z}_{2}\right)+\text { reg. },
\end{aligned}
$$

where "reg." stands for regular terms and where the OPEs between $\mathscr{J}^{a}(z)$ and $\overline{\mathscr{J}}^{b}(\bar{z})$ are purely regular. Note that (3.9) defines two independent non-abelian current algebras with structure constants $f^{a b}{ }_{c} \simeq H^{a b}{ }_{c}$. The only difference to the usual expressions is an opposite relative sign for $H^{a b}{ }_{c}$ between the holomorphic and anti-holomorphic parts. 
Next, we turn to the energy-momentum tensor. Up to linear order in the flux we find

$$
\mathscr{T}(z)=\frac{1}{\alpha^{\prime}} \delta_{a b}: \mathscr{J}^{a} \mathscr{J}^{b}:(z), \quad \bar{T}(\bar{z})=\frac{1}{\alpha^{\prime}} \delta_{a b}: \overline{\mathscr{J}}^{a} \overline{\mathscr{J}}^{b}:(\bar{z}),
$$

and the anti-symmetry of $H$ implies that the OPEs of two energy-momentum tensors take the form

$$
\begin{aligned}
& \mathscr{T}\left(z_{1}\right) \mathscr{T}\left(z_{2}\right)=\frac{c / 2}{\left(z_{1}-z_{2}\right)^{4}}+\frac{2 \mathscr{T}\left(z_{2}\right)}{\left(z_{1}-z_{2}\right)^{2}}+\frac{\partial \mathscr{T}\left(z_{2}\right)}{z_{1}-z_{2}}+\text { reg. }, \\
& \overline{\mathscr{T}}\left(\bar{z}_{1}\right) \overline{\mathscr{T}}\left(\bar{z}_{2}\right)=\frac{c / 2}{\left(\bar{z}_{1}-\bar{z}_{2}\right)^{4}}+\frac{2 \overline{\mathscr{T}}\left(z_{2}\right)}{\left(\bar{z}_{1}-\bar{z}_{2}\right)^{2}}+\frac{\partial \overline{\mathscr{T}}\left(z_{2}\right)}{\bar{z}_{1}-\bar{z}_{2}}+\text { reg. },
\end{aligned}
$$

with $\mathscr{T}\left(z_{1}\right) \overline{\mathscr{T}}\left(\bar{z}_{2}\right)$ regular. We therefore find two copies of the Virasoro algebra with the same central charge $c$ as for the free theory. Moreover, using (3.9) and again the anti-symmetry of $H$, one can show that the fields $\mathscr{J}^{a}(z)$ and $\overline{\mathscr{J}}^{a}(\bar{z})$ are primary of conformal dimension $(1,0)$ and $(0,1)$ with respect to $\mathscr{T}(z)$ and $\bar{T}(\bar{z})$; and so they are indeed non-abelian currents.

Basic three-point function Let us now define fields $\mathscr{X}^{a}(z, \bar{z})$ as the integrals of (3.6). In particular, we write

$$
\mathscr{J}^{a}(z)=i \partial \mathscr{X}^{a}(z, \bar{z}), \quad \quad \bar{J}^{a}(\bar{z})=i \bar{\partial} \mathscr{X}^{a}(z, \bar{z})
$$

The three-point function of three $\mathscr{X}^{a}$ up to first order in the $H$-flux can then be obtained by integrating the corresponding correlators (3.7). For that purpose, we introduce the Rogers dilogarithm $L(z)$ which is defined in terms of the usual dilogarithm $\mathrm{Li}_{2}(z)$ as follows

$$
L(z)=\mathrm{Li}_{2}(z)+\frac{1}{2} \log (z) \log (1-z) .
$$

For the correlator of three fields $\mathscr{X}^{a}(z, \bar{z})$ one obtains (see [17] for more details)

$$
\left\langle\mathscr{X}^{a}\left(z_{1}, \bar{z}_{1}\right) \mathscr{X}^{b}\left(z_{2}, \bar{z}_{2}\right) \mathscr{X}^{c}\left(z_{3}, \bar{z}_{3}\right)\right\rangle=\frac{\alpha^{\prime 2}}{12} H^{a b c}\left[L\left(\frac{z_{12}}{z_{13}}\right)+L\left(\frac{z_{23}}{z_{21}}\right)+L\left(\frac{z_{13}}{z_{23}}\right)-\text { c.c. }\right],
$$

where "c.c." stands for complex conjugation. To simplify our notation for the following, let us recall from (3.1) the relation between the flux $H$ and the flux parameter $\theta$, that is $\theta^{a b c}=\frac{\alpha^{\prime 2}}{12} H^{a b c}$, and let us introduce

$$
\mathscr{L}(z)=L(z)+L\left(1-\frac{1}{z}\right)+L\left(\frac{1}{1-z}\right) .
$$

The correlation function (3.14) of three fields $\mathscr{X}^{a}(z, \bar{z})$ in the $H$-flux background can then be written as

$$
\left\langle\mathscr{X}^{a}\left(z_{1}, \bar{z}_{1}\right) \mathscr{X}^{b}\left(z_{2}, \bar{z}_{2}\right) \mathscr{X}^{c}\left(z_{3}, \bar{z}_{3}\right)\right\rangle=\theta^{a b c}\left[\mathscr{L}\left(\frac{z_{12}}{z_{13}}\right)-\mathscr{L}\left(\frac{\bar{z}_{12}}{\bar{z}_{13}}\right)\right] .
$$

Vertex operator for the tachyon We now define vertex operators. In analogy to the free theory of a closed string without $H$-flux, which in a compact space can have momentum $p_{a}$ and winding $w^{b}$, we define left- and right-moving momenta $k_{L / R}$ as

$$
k_{L}^{a}=p^{a}+\frac{w^{a}}{\alpha^{\prime}}, \quad \quad k_{R}^{a}=p^{a}-\frac{w^{a}}{\alpha^{\prime}} .
$$


The vertex operator for the perturbed theory should then be written in the following way

$$
\mathscr{V}(z, \bar{z})=: \exp \left(i k_{L} \cdot \mathscr{X}_{L}+i k_{R} \cdot \mathscr{X}_{R}\right):,
$$

where we employ the short-hand notation $k_{L} \cdot \mathscr{X}_{L}=k_{L a} \mathscr{X}_{L}^{a}, \ldots$ : denotes normal ordering and the left- and right-moving fields $\mathscr{X}_{L / R}^{a}$ are obtained via integration of the currents. Now, recall that in the free theory the tachyon vertex operator is a primary field of conformal dimension $(h, \bar{h})=\left(\frac{\alpha^{\prime}}{4} k_{L}^{2}, \frac{\alpha^{\prime}}{4} k_{R}^{2}\right)$, and in covariant quantization of the bosonic string physical states are given by primary fields of conformal dimension $(h, \bar{h})=(1,1)$. In the deformed theory, we also require that vertex operators $\mathscr{V}(z, \bar{z})$ are primary with respect to $\mathscr{T}(z)$ and $\bar{T}(\bar{z})$ which is not guaranteed a priori. However, it is again the anti-symmetry of $H$ which implies

$$
\begin{aligned}
& \mathscr{T}\left(z_{1}\right) \mathscr{V}\left(z_{2}, \bar{z}_{2}\right)=\frac{1}{\left(z_{1}-z_{2}\right)^{2}} \frac{\alpha^{\prime} k_{L} \cdot k_{L}}{4} \mathscr{V}\left(z_{2}, \bar{z}_{2}\right)+\frac{1}{z_{1}-z_{2}} \partial \mathscr{V}\left(z_{2}, \bar{z}_{2}\right)+\text { reg. } \\
& \overline{\mathscr{T}}\left(\bar{z}_{1}\right) \mathscr{V}\left(z_{2}, \bar{z}_{2}\right)=\frac{1}{\left(\bar{z}_{1}-\bar{z}_{2}\right)^{2}} \frac{\alpha^{\prime} k_{R} \cdot k_{R}}{4} \mathscr{V}\left(z_{2}, \bar{z}_{2}\right)+\frac{1}{\bar{z}_{1}-\bar{z}_{2}} \bar{\partial} \mathscr{V}\left(z_{2}, \bar{z}_{2}\right)+\text { reg. }
\end{aligned}
$$

Thus, the vertex operator (3.18) is primary and has the correct conformal dimension, and is therefore a physical quantum state of the deformed theory. However, as it is explained in more detail in [17], in order for the tachyon vertex operator to carry momenta $\left(k_{L}, k_{R}\right)$ we have to require

$$
0=H^{a}{ }_{b c} k_{L}^{b} k_{R}^{c} \simeq H^{a}{ }_{b c} p^{b} w^{c} \simeq[\vec{p} \times \vec{w}]^{a} .
$$

\subsection{T-duality}

As expected from the string equations of motion (3.3), in the last subsection we have found a conformal field theory which describes the sigma model for a flat metric and constant $H$-flux up to linear order. However, we are also interested in backgrounds T-dual to the $H$-flux configuration. On the level of the CFT, T-duality is usually realized as a reflection on the right-moving coordinates. Since the corrected fields $\mathscr{X}^{a}(z, \bar{z})$ still admit a split into a holomorphic and an anti-holomorphic piece, we define T-duality on the world-sheet along a direction $\mathscr{X}^{a}$ as

$$
\begin{array}{lll}
\mathscr{X}_{L}^{a}(z) & \text { T-duality } & +\mathscr{X}_{L}^{a}(z), \\
\mathscr{X}_{R}^{a}(\bar{z}) & & -\mathscr{X}_{R}^{a}(\bar{z}) .
\end{array}
$$

Clearly, for the currents this implies a similar action, and so the "structure constants" $H^{a b}{ }_{c}$ in the anti-holomorphic OPE (3.9) receive an additional minus sign when performing a T-duality transformation.

In the next subsection, we compute scattering amplitudes for tachyon vertex operators in the $H$-flux background. There we allow for momentum and winding along the directions of our threedimensional (compact) space specified by (3.1). However, in the T-dual backgrounds we are particularly interested in pure momentum scattering, as from there one would derive the low-energy effective action as a derivative expansion. Now, above we have mentioned that a tachyon vertex operator $\mathscr{V}(z, \bar{z})$ indeed corresponds to a physical state provided that $\vec{p} \times \vec{w}=\overrightarrow{0}$. This has the following implications (see again [17] for further details): 
- The effective field theory for tachyons in the $H$-flux background is expected to be reliably computable from scattering amplitudes of pure momentum tachyons, since in this case the constraint (3.20) is satisfied.

- For backgrounds with geometric flux, pure momentum scattering is related to the scattering of for instance $\left(p_{1}, p_{2}, \omega_{3}\right)$ modes in the $H$-flux background. However, in this case $\vec{\omega} \times \vec{p} \neq \overrightarrow{0}$ and so we can not reliably employ the vertex operator for the tachyon in our present approach. A similar situation occurs for pure momentum scattering in the $Q$-flux background.

- For the case of $R$-flux we can again reliably compute the scattering amplitudes for pure momentum tachyons. By T-duality, they are related to the scattering of pure winding states in the $H$-flux background for which (3.20) is satisfied.

Therefore, in the following we focus on backgrounds with $H$ - and $R$-flux.

\subsection{Tachyon scattering amplitudes}

In this subsection, we compute scattering amplitudes of the tachyon vertex operators (3.18) and extract a tri-product.

Three-tachyon amplitude As discussed above, we focus on a compact three-dimensional space and therein we are interested in pure momentum $\left(p_{1}, p_{2}, p_{3}\right)$ or pure winding $\left(w_{1}, w_{2}, w_{3}\right)$ state scattering, where the latter is related by three T-dualities to pure momentum scattering in the $R$ flux background. We therefore consider vertex operators of form

$$
\begin{aligned}
& \mathscr{V}_{i}^{H} \equiv \mathscr{V}_{p_{i}}\left(z_{i}, \bar{z}_{i}\right)=: \exp \left(i p_{i} \cdot \mathscr{X}\left(z_{i}, \bar{z}_{i}\right)\right): \\
& \mathscr{V}_{i}^{R} \equiv \mathscr{V}_{w_{i}}\left(z_{i}, \bar{z}_{i}\right)=: \exp \left(i w_{i} \cdot \widetilde{\mathscr{X}}\left(z_{i}, \bar{z}_{i}\right)\right):,
\end{aligned}
$$

where $\widetilde{\mathscr{X}}=\mathscr{X}_{L}-\mathscr{X}_{R}$. Note that here and in the following we employ the short hand notation $\mathscr{V}_{i}^{H / R}$ and $V_{i}^{H / R}$ for the vertex operators of the perturbed and free theory, respectively. Furthermore, since we can consider $\mathscr{V}_{i}^{R}$ as the momentum vertex operator in the $R$-flux background, in the following we set $\left.\left.w\right|_{H} \rightarrow p\right|_{R}$.

Next, in string theory one has to work with vertex operators integrated over the world-sheet. We therefore define

$$
\mathscr{T}_{i}^{H / R}=\int d^{2} z^{\mathscr{V}_{i}^{H / R}}
$$

Taking into account the freedom to fix three points on the world-sheet via the $S L(2, \mathbb{C})$ symmetry, the three-tachyon scattering amplitude is then given by

$$
\left\langle\mathscr{T}_{1} \mathscr{T}_{2} \mathscr{T}_{3}\right\rangle^{H / R}=\int \prod_{i=1}^{3} d^{2} z_{i} \delta^{(2)}\left(z_{i}-z_{i}^{0}\right)\left|z_{12} z_{13} z_{23}\right|^{2}\left\langle\mathscr{V}_{1} \mathscr{V}_{2} \mathscr{V}_{3}\right\rangle^{H / R}
$$

where we have put the superscript indicating the $H$ - and $R$-flux background outside the bracket in order to shorten the notation. Using (3.16), for the correlator of three vertex operators one obtains

$$
\left\langle\mathscr{V}_{1} \mathscr{V}_{2} \mathscr{V}_{3}\right\rangle^{H / R}=\frac{\delta\left(p_{1}+p_{2}+p_{3}\right)}{\left|z_{12} z_{13} z_{23}\right|^{2}} \exp \left[-i \theta^{a b c} p_{1, a} p_{2, b} p_{3, c}\left[\mathscr{L}\left(\frac{z_{12}}{z_{13}}\right) \mp \mathscr{L}\left(\frac{\bar{z}_{12}}{\bar{z}_{13}}\right)\right]\right]_{\theta},
$$


where $[\ldots]_{\theta}$ indicates that the result is valid only up to linear order in $\theta$. Note that the upper sign corresponds to the case of $H$-flux and the lower to the case of $R$-flux. The full scattering amplitude then becomes

$$
\begin{aligned}
\left\langle\mathscr{T}_{1} \mathscr{T}_{2} \mathscr{T}_{3}\right\rangle^{H / R}=\int \prod_{i=1}^{3} d^{2} z_{i} \delta^{(2)}\left(z_{i}-z_{i}^{0}\right) \delta\left(p_{1}+p_{2}+p_{3}\right) \times \\
\exp \left[-i \theta^{a b c} p_{1, a} p_{2, b} p_{3, c}\left[\mathscr{L}\left(\frac{z_{12}}{z_{13}}\right) \mp \mathscr{L}\left(\frac{\bar{z}_{12}}{\bar{z}_{13}}\right)\right]\right]_{\theta} .
\end{aligned}
$$

Permutations Let us now study the behavior of (3.25) under permutations of the vertex operators $\mathscr{V}_{i}^{H / R}$. Before applying momentum conservation, the three-tachyon amplitude for a permutation $\sigma$ can be computed using properties of the Rogers dilogarithm. With $\varepsilon=-1$ for $H$-flux and $\varepsilon=+1$ for the case of $R$-flux, one finds

$$
\left\langle\mathscr{V}_{\sigma(1)} \mathscr{V}_{\sigma(2)} \mathscr{V}_{\sigma(3)}\right\rangle^{\varepsilon}=\exp \left[i\left(\frac{1+\varepsilon}{2}\right) \eta_{\sigma} \pi^{2} \theta^{a b c} p_{1, a} p_{2, b} p_{3, c}\right]\left\langle\mathscr{V}_{1} \mathscr{V}_{2} \mathscr{V}_{3}\right\rangle^{\varepsilon},
$$

where in addition $\eta_{\sigma}=1$ for an odd permutation and $\eta_{\sigma}=0$ for an even one. Thus, for the $R$-flux background a non-trivial phase may appear which we have established up to linear order in the flux. As will be discussed in more detail in section 3.4, the phase in (3.27) can be recovered from a three-product on the space of functions $V_{p_{n}}(x)=\exp \left(i p_{n} \cdot x\right)$, which can be defined as

$$
V_{p_{1}}(x) \triangle V_{p_{2}}(x) \Delta V_{p_{3}}(x) \stackrel{\text { def }}{=} \exp \left(-i \frac{\pi^{2}}{2} \theta^{a b c} p_{1, a} p_{2, b} p_{3, c}\right) V_{p_{1}+p_{2}+p_{3}}(x) .
$$

However, in correlation functions operators are understood to be radially ordered and so changing the order of operators should not change the form of the amplitude. This is known as crossing symmetry which is one of the defining properties of a CFT and thus should also be satisfied here. In the case of the $R$-flux background, this is reconciled by applying momentum conservation leading to

$$
p_{1, a} p_{2, b} p_{3, c} \theta^{a b c}=0 \quad \text { for } \quad p_{3}=-p_{1}-p_{2} .
$$

Therefore, scattering amplitudes of three tachyons do not receive any corrections at linear order in $\theta$ both for the $H$ - and $R$-flux

$$
\left\langle\mathscr{T}_{1} \mathscr{T}_{2} \mathscr{T}_{3}\right\rangle^{H / R}=\delta\left(p_{1}+p_{2}+p_{3}\right) .
$$

Four- and N-tachyon amplitudes We now want to detect phases possibly appearing for the product of $N$ closed string tachyon vertex operators. But before we consider the general case, let us start with the amplitude of four tachyons. Up to linear order in $\theta$ we obtain

$$
\left\langle\mathscr{V}_{1} \mathscr{V}_{2} \mathscr{V}_{3} \mathscr{V}_{4}\right\rangle^{H / R}=\left\langle V_{1} V_{2} V_{3} V_{4}\right\rangle_{0}^{H / R} \exp \left[-i \theta^{a b c} \sum_{1 \leq i<j<k \leq 4} p_{i, a} p_{j, b} p_{k, c}\left[\mathscr{L}\left(\frac{z_{i j}}{z_{i k}}\right) \mp \mathscr{L}\left(\frac{\bar{z}_{i j}}{\bar{z}_{i k}}\right)\right]\right]_{\theta} .
$$

Again, the difference between $H$ - and $R$-flux is given by the sign between the holomorphic and the anti-holomorphic contribution, and the four-point function $\left\langle V_{1} V_{2} V_{3} V_{4}\right\rangle_{0}^{H / R}$ is just the one from the free theory. We can now determine the behavior of the amplitude under a permutation of the 
vertex operators. Prior to using momentum conservation, we again find momentum dependent phase factors. Analogous to the three-tachyon amplitude, these arise in the case of $R$-flux and can be described as resulting from a deformed four-product of the form

$$
\begin{aligned}
& V_{p_{1}}(x) \triangle_{4} V_{p_{2}}(x) \triangle_{4} V_{p_{3}}(x) \triangle_{4} V_{p_{4}}(x) \stackrel{\text { def }}{=} \exp \left[-i \frac{\pi^{2}}{2} \theta^{a b c}\left(p_{1, a} p_{2, b} p_{3, c}\right.\right. \\
&\left.\left.+p_{1, a} p_{2, b} p_{4, c}+p_{1, a} p_{3, b} p_{4, c}+p_{2, a} p_{3, b} p_{4, c}\right)\right] V_{\sum p_{i}}(x) .
\end{aligned}
$$

However, employing momentum conservation, one can show that this phase becomes trivial so that the four-tachyon amplitude is indeed crossing symmetric.

This computation for four tachyons can straightforwardly be generalized to higher $N$-tachyon amplitudes. The phase factors appearing when permuting two vertex operators for the case of the $R$-flux background can then be encoded in a deformed $N$-product of the form

$$
V_{p_{1}}(x) \triangle_{N} \ldots \triangle_{N} V_{p_{N}}(x) \stackrel{\text { def }}{=} \exp \left(-i \frac{\pi^{2}}{2} \theta^{a b c} \sum_{1 \leq i<j<k \leq N} p_{i, a} p_{j, b} p_{k, c}\right) V_{\sum p_{i}}(x) .
$$

The phase becomes again trivial after employing momentum conversation so that all $N$-tachyon correlators are crossing symmetric. This signals that the basic principle of perturbative closed string theory, namely conformal field theory, seems to be compatible with non-geometric backgrounds for which the $N$-product of functions is deformed by (3.33).

\subsection{A tri-product}

In this section, we show that the relative phase factors of tachyon correlation functions can be rephrased in terms of a generalization of the Moyal-Weyl star-product, which we call a tri-product.

Tri-product In particular, the phase appearing in the three-point correlator (3.27) indicates that we can define a three-product of functions $f(x)$ in the following way

$$
\left.f_{1}(x) \triangle f_{2}(x) \triangle f_{3}(x) \stackrel{\text { def }}{=} \exp \left(\frac{\pi^{2}}{2} \theta^{a b c} \partial_{a}^{x_{1}} \partial_{b}^{x_{2}} \partial_{c}^{x_{3}}\right) f_{1}\left(x_{1}\right) f_{2}\left(x_{2}\right) f_{3}\left(x_{3}\right)\right|_{x}
$$

where we used the notation $\left.()\right|_{x}=\left.()\right|_{x_{1}=x_{2}=x_{3}=x}$. Choosing $f_{n}(x)=\exp \left(i p_{n} \cdot x\right)$ we obtain formula (3.28), which after integration over $x$ gives

$$
\begin{aligned}
\int d^{3} x f_{1}(x) \triangle f_{2}(x) \triangle f_{3}(x) & =\exp \left(-i \frac{\pi^{2}}{2} \theta^{a b c} p_{1, a} p_{2, b} p_{3, c}\right) \delta\left(p_{1}+p_{2}+p_{3}\right) \\
& =\int d^{3} x f_{1}(x) f_{2}(x) f_{3}(x)
\end{aligned}
$$

Note that (3.34) is precisely the three-product (2.26). Indeed, with $P^{3}$ denoting the permutation group of three elements, the three-bracket for the coordinates $x^{a}$ can then be re-derived as the completely anti-symmetrized sum of three-products

$$
\left[x^{a}, x^{b}, x^{c}\right]=\sum_{\sigma \in P^{3}} \operatorname{sign}(\sigma) x^{\sigma(a)} \triangle x^{\sigma(b)} \triangle x^{\sigma(c)}=3 \pi^{2} \theta^{a b c} .
$$


N-product Next, we consider the $N$-tachyon amplitude and the phase appearing in equation (3.33). This motivates us to define the $N$-product

$$
\left.f_{1}(x) \triangle_{N} f_{2}(x) \Delta_{N} \ldots \Delta_{N} f_{N}(x) \stackrel{\text { def }}{=} \exp \left[\frac{\pi^{2}}{2} \theta^{a b c} \sum_{1 \leq i<j<k \leq N} \partial_{a}^{x_{i}} \partial_{b}^{x_{j}} \partial_{c}^{x_{k}}\right] f_{1}\left(x_{1}\right) f_{2}\left(x_{2}\right) \ldots f_{N}\left(x_{N}\right)\right|_{x},
$$

which is the closed string generalization of the open string non-commutative product (1.4). This completely defines the new tri-product, which satisfies the relation

$$
f_{1} \triangle_{N} f_{2} \triangle_{N} \ldots \triangle_{N} f_{N-1} \triangle_{N} 1=f_{1} \triangle_{N-1} \ldots \triangle_{N-1} f_{N-1} .
$$

Specializing this expression to $N=3$ gives

$$
f_{1} \triangle_{2} f_{2}=f_{1} \triangle_{3} f_{2} \triangle_{3} 1=f_{1} \cdot f_{2}
$$

which just means that the tri-product of two functions is the usual commutative point-wise product. However, there are two main differences compared to the open-string case.

- For the open string the star $N$-product was related to successive application of the usual Moyal-Weyl bi-product. This simplifying behavior is not true for the tri-product, i.e. the $N$-products $\Delta_{N}$ can not be related to successive applications of the three-product $\triangle=\Delta_{3}$. This means that one does not only have to specify a deformed product of three functions (with the rest following), but has to specify a definition for a deformed product of any number of functions.

- In contrast to the open string case, the effect of the tri-product in integrals vanishes, i.e.

$$
\int d^{n} x f_{1}(x) \Delta_{N} f_{2}(x) \Delta_{N} \ldots \Delta_{N} f_{N}(x)=\int d^{n} x f_{1}(x) f_{2}(x) \ldots f_{N}(x) .
$$

In other words, the difference between the tri-product and the ordinary product is a total derivative. Thus, closed strings can consistently be defined on non-associative backgrounds since in string scattering amplitudes its effect vanishes. 


\section{Summary}

In these proceedings, we have summarized recent work on non-commutativity in closed string theory which appeared in [6] and [17]. In particular, motivated by results from the open-string sector, we have illustrated two approaches to study non-commutative behavior for closed strings.

The first approach mimics the computation of the commutator of two open-string coordinates. However, in the case of a closed string one should consider a cyclic double commutator involving three instead of two fields. If such an expression is non-vanishing, it not only indicates a noncommutative but also a non-associative structure. Furthermore, we illustrated that in order to obtain a non-trivial result, background fluxes have to be non-vanishing. These can be ordinary $H$-fluxes or geometric fluxes, but also non-geometric $Q$ - and $R$-fluxes. In section 2 , we have computed the cyclic double commutator shown in equation (2.26) and found it to be non-vanishing for a background with $R$-flux, and therefore indicating a non-associative structure.

The second approach to non-commutativity considered here is to compute correlation functions of vertex operators. Since the non-commutative effect obtained in (2.26) is linear in the flux parameter, we studied a conformal field theory up to linear order in the background flux. Defining then a tachyon vertex operator in this CFT and computing correlation functions thereof, we obtained a phase factor which encodes the flux dependence (up to linear order). From this factor we motivated and studied a tri-product (3.34) which captures non-commutative and non-associative effects.

The direction for future work is to make the non-commutative and non-associative structure more apparent. For instance, the relation between $R$-flux and the non-vanishing three-bracket (2.26) leading to a non-associative behavior has to be understood better. Furthermore, properties of the tri-product (3.34) have to be studied, which is needed to eventually formulate an effective theory using that product.

Acknowledgements We would like thank Ralph Blumenhagen, Andreas Deser, Dieter Lüst and Felix Rennecke for collaboration and discussions on the material presented in this talk. We are also grateful to the organizers of the "Workshop on Noncommutative Field Theory and Gravity" at the Corfu Summer Institute for the opportunity to present this material. The author is supported by the Netherlands Organization for Scientific Research (NWO) under the VICI grant 680-47-603. 


\section{References}

[1] A. Connes, M. R. Douglas, and A. S. Schwarz, "Noncommutative geometry and matrix theory: Compactification on tori," JHEP 02 (1998) 003, hep-th / 9711162.

[2] C.-S. Chu and P.-M. Ho, "Noncommutative open string and D-brane," Nucl. Phys. B550 (1999) 151-168, hep-th/9812219.

[3] V. Schomerus, "D-branes and deformation quantization,” JHEP 06 (1999) 030, hep-th/ 9903205.

[4] N. Seiberg and E. Witten, "String theory and noncommutative geometry," JHEP 09 (1999) 032 , hep-th/9908142.

[5] K. Dasgupta, G. Rajesh, D. Robbins, and S. Sethi, "Time dependent warping, fluxes, and NCYM," JHEP 0303 (2003) 041, hep-th/ 0302049.

[6] R. Blumenhagen and E. Plauschinn, "Nonassociative Gravity in String Theory?," J.Phys.A A44 (2011) 015401, 1010.1263.

[7] E. Witten, "Nonabelian bosonization in two dimensions," Commun. Math. Phys. 92 (1984) 455-472.

[8] K. Dasgupta, G. Rajesh, and S. Sethi, "M theory, orientifolds and G - flux," JHEP 9908 (1999) 023, hep-th/9908088.

[9] J. Shelton, W. Taylor, and B. Wecht, "Nongeometric Flux Compactifications," JHEP 10 (2005) 085, hep-th/0508133.

[10] P. Bouwknegt, K. Hannabuss, and V. Mathai, "Nonassociative tori and applications to T-duality," Commun. Math. Phys. 264 (2006) 41-69, hep-th/ 0412092.

[11] P. Bouwknegt, K. Hannabuss, and V. Mathai, "T-duality for principal torus bundles and dimensionally reduced Gysin sequences,” Adv. Theor. Math. Phys. 9 (2005) 749-773, hep-th / 0412268.

[12] D. Lüst, "T-duality and closed string non-commutative (doubled) geometry," JHEP 1012 (2010) 084, 1010.1361 .

[13] C. Saemann and R. J. Szabo, “Groupoid Quantization of Loop Spaces,” 1203.5921.

[14] L. Cornalba and R. Schiappa, "Nonassociative star product deformations for D-brane worldvolumes in curved backgrounds," Commun. Math. Phys. 225 (2002) 33-66, hep-th/ 0101219.

[15] M. Herbst, A. Kling, and M. Kreuzer, "Star products from open strings in curved backgrounds," JHEP 09 (2001) 014, hep-th/ 0106159.

[16] C. Condeescu, I. Florakis, and D. Lüst, “Asymmetric Orbifolds, Non-Geometric Fluxes and Non-Commutativity in Closed String Theory," 1202.6366.

[17] R. Blumenhagen, A. Deser, D. Lüst, E. Plauschinn, and F. Rennecke, "Non-geometric Fluxes, Asymmetric Strings and Nonassociative Geometry," J.Phys.A A44 (2011) 385401, 1106.0316.

[18] R. Blumenhagen, "Nonassociativity in String Theory," 1112.4611.

[19] D. Andriot, M. Larfors, D. Lüst, and P. Patalong, “A ten-dimensional action for non-geometric fluxes," JHEP 1109 (2011) 134, 1106.4015.

[20] D. Andriot, O. Hohm, M. Larfors, D. Lüst, and P. Patalong, “A geometric action for non-geometric fluxes," 1202.3060 .4 pages.

[21] P. Patalong, "Non-geometric Q-flux in ten dimensions," 1203.5127. 
[22] R. Blumenhagen, A. Deser, E. Plauschinn, and F. Rennecke, "Palatini-Lovelock-Cartan Gravity Bianchi Identities for Stringy Fluxes," 1202.4934.

[23] R. Blumenhagen, A. Deser, E. Plauschinn, and F. Rennecke, “to appear,".

[24] D. Gepner and E. Witten, “String Theory on Group Manifolds," Nucl. Phys. B278 (1986) 493.

[25] E. Braaten, T. L. Curtright, and C. K. Zachos, "Torsion and Geometrostasis in Nonlinear Sigma Models," Nucl. Phys. B260 (1985) 630. 